\title{
Transport of energy in dissipative advection phenomena
}

Manuel Núñez

Citation: Journal of Mathematical Physics 44, 1218 (2003); doi: 10.1063/1.1540237

View online: https://doi.org/10.1063/1.1540237

View Table of Contents: http://aip.scitation.org/toc/jmp/44/3

Published by the American Institute of Physics

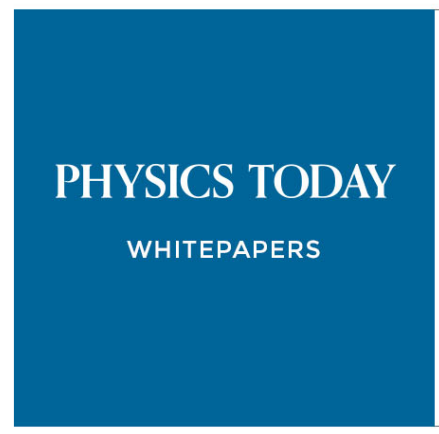

MANAGER'S GUIDE

Accelerate R\&D with

Multiphysics Simulation

\section{READ NOW}

PRESENTED BY $\checkmark \subset \mathrm{MSOL}$ 


\section{Transport of energy in dissipative advection phenomena}

Manuel Núñez ${ }^{\text {a) }}$

Departamento de Análisis Matemático, Universidad de Valladolid, 47005 Valladolid, Spain

(Received 4 November 2002; accepted 26 November 2002)

A study of the distribution of energy among the different scales is performed for several systems in fluid mechanics, including the Navier-Stokes, magnetohydrodynamics and active scalars equations. It is found that all these systems possess a common structure which enables us to deduce how the energy introduced by the forcing is transferred to the scales present in the flow. It is also shown that in special cases an energy cascade will occur. The limits of this method are also considered. (C) 2003 American Institute of Physics. [DOI: 10.1063/1.1540237]

\section{INTRODUCTION}

It is well known that the energy injected into a fluid by some forcing will be distributed among all the scales of the flow. In a general sense this is true for all phenomena governed by nonlinear equations: for every decomposition of the solution in different modes representing the scales, the nonlinearity will make interact different modes so that they do not evolve independently. The details of how this interaction occurs depend essentially on the particular equation modeling the phenomenon. However, for certain equations, prevalent in several processes in fluid mechanics, the transfer of energy may be studied with some generality. These equations could be called dissipative advection phenomena by the two terms of their expression:

$$
\frac{\partial w}{\partial t}=A w+T w+f,
$$

where $w$ is the magnitude under study. It is assumed that the solution of our initial value problem exists for all time, and that $w(t)$ belongs to a certain Hilbert space $H . A$ is a linear self-adjoint dissipative operator; it is defined in a dense domain

$$
A: D(A) \rightarrow H,
$$

and it satisfies, for some constant $\alpha>0$ and all $w \in D(A)$,

$$
-(A w, w) \geqslant \alpha\|w\|^{2} .
$$

Thus the whole spectrum of $A$ is contained in $(-\infty,-\alpha]$. For simplicity purposes we will assume that $H$ is separable and the spectrum of $A$ discrete, although it is not really necessary. $T$ is a generally nonlinear and time-dependent operator densely defined in $H$, satisfying the orthogonality property:

$$
(T w, w)=0,
$$

for every $w$ in the domain of $T$. A represents the dissipation and $T$ the advective effect in the evolution of $w$. As we will see, condition (3) means that the energy injected by the forcing $f$ will be distributed without loss by this advective term among all scales, while dissipation acts in a different way on each of them.

${ }^{a)}$ Electronic mail: mnjmhd@am.uva.es 
The present article represents a simplification of the arguments advanced in Ref. 1 for the study of hydrodynamics turbulence. The Navier-Stokes case is analyzed there in more generality and depth, without making use of some of our hypotheses concerning uniqueness of the solution, which in fact are not proved in general. While it is probably overoptimistic to think that Ref. 1 answers all the questions on the origin and behavior of cascades in turbulence, it represents a valuable attempt to rigorize a classical and difficult problem. The method developed there is not difficult and we have been able to extend its range beyond the original hydrodynamic problem: in addition to the Navier-Stokes equations, the magnetohydrodynamics (MHD) system possesses also the required form, along with several equations describing passive and active scalars. Among the latter we may mention the vorticity in plane flows, the magnetic potential in two-dimensional plasmas, the temperature in Bénard convection, the salinity in oceanic waters and other magnitudes relevant in geophysical phenomena.

In a limit case we can prove something similar to energy cascades in the sense of Kolmogorov: energy is transferred without loss to smaller (direct cascade) or larger scales (inverse cascade) in a form independent of $A$, i.e., of viscosity. ${ }^{2}$ However, the assumptions needed to prove this are probably excessive and the fact that there is no discernible influence of the space dimension, unlike what is experimentally known, seems to indicate that physical cascades have their own phenomenology not covered by this case.

\section{THE MAIN RESULTS}

Let $\left(e_{n}\right)$ be a basis of $H$ formed by eigenfunctions of $A,-A e_{n}=\lambda_{n} e_{n}$, with $\lambda_{1}=\alpha, \lambda_{1}$ $\leqslant \lambda_{2} \leqslant \cdots$. We will consider that the scales of the problem are given by the orthogonal decomposition $w=\Sigma\left(w, e_{n}\right) e_{n}$, so that a large-scale function is one limited to the lower eigenfunctions. When, as usual, $A$ is essentially the Laplacian, certainly the higher eigenfunctions are more irregular than the lower ones. In particular, if $H$ is formed by periodic functions, the basis is formed by the trigonometric functions $\mathbf{x} \rightarrow \exp (i \mathbf{k} \cdot \mathbf{x})$ and the spectral decomposition is the Fourier one, so that the concept coincides with the classical one.

The energy estimates are classical: by making the scalar product of (1) with $w$,

$$
\frac{1}{2} \frac{\partial}{\partial t}(w, w)-(A w, w)=(T w, w)+(f, w)=(f, w) .
$$

Thus

$$
\frac{1}{2} \frac{d}{d t}\|w\|^{2}-(A w, w)=(f, w) \leqslant\|f\|\|w\| \leqslant \frac{1}{2 \alpha}\|f\|^{2}+\frac{\alpha}{2}\|w\|^{2} .
$$

Since $-(A w, w) \geqslant \alpha\|w\|^{2}$,

$$
\frac{d}{d t}\|w\|^{2}+\alpha\|w\|^{2} \leqslant \frac{1}{\alpha}\|f\|^{2}
$$

so that

$$
\|w(t)\|^{2} \leqslant\|w(0)\|^{2} e^{-\alpha t}+\frac{1}{\alpha} \int_{0}^{t} e^{-\alpha(t-s)}\|f(s)\|^{2} \mathrm{~d} s,
$$

which is bounded in particular if $\|f\|$ is bounded for all time: e.g., if it does not depend on time. In the absence of forcing the solution tends exponentially to zero. We will only need to assume

$$
\lim _{t \rightarrow \infty} \frac{1}{T}\|w(T)\|^{2}=0
$$


which certainly happens if $w$ remains bounded. Our remaining hypotheses are as follows: let us denote by \langle\rangle the time mean of a magnitude:

$$
\langle g\rangle=\lim _{T \rightarrow \infty} \frac{1}{T} \int_{0}^{T} g(s) \mathrm{d} s,
$$

provided it exists. We will assume that the time mean of all the magnitudes occurring in Eqs. (11)-(16) exists. This is not an unreasonable hypothesis, since turbulent phenomena are usually considered decaying or statistically stationary.

Let $H_{1}$ be the finite-dimensional subspace of $H$ whose basis is $\left\{e_{k}, e_{k+1}, \ldots, e_{l}\right\}$. We will consider that $H_{1}$ is the space of some intermediate range of scales, called the injection range because we will assume that $f$ lies within this range for all time; if $f$ is large-scale, then $k=1$. Let $H_{2}$ be the subspace orthogonal to $H_{1}$, a Hilbert basis of whom is formed by the remaining $e_{j}$. Let $w=w_{1}+w_{2}$ be the orthogonal decomposition of an element of $H$ in the subspaces $H_{1}$ and $H_{2}$. The condition $(T w, w)=0$ means therefore

$$
\left(T w, w_{2}\right)=-\left(T w, w_{1}\right) .
$$

By taking the scalar product of (1) with $w_{1}$ and $w_{2}$ we obtain, analogously to (4),

$$
\begin{gathered}
\frac{1}{2} \frac{d}{d t}\left\|w_{1}\right\|^{2}-\left(A w_{1}, w_{1}\right)=\left(T w, w_{1}\right)+\left(f, w_{1}\right), \\
\frac{1}{2} \frac{d}{d t}\left\|w_{2}\right\|^{2}-\left(A w_{2}, w_{2}\right)=\left(T w, w_{2}\right)=-\left(T w, w_{1}\right),
\end{gathered}
$$

since $\left(f, w_{2}\right)=0$. We also have

$$
\left\langle\frac{d}{d t}\left\|w_{i}\right\|^{2}\right\rangle=\lim _{T \rightarrow \infty} \frac{1}{T}\left(\left\|w_{i}(T)\right\|^{2}-\left\|w_{i}(0)\right\|^{2}\right)=0
$$

and therefore

$$
\begin{gathered}
\left\langle\left(-A w_{1}, w_{1}\right)\right\rangle=\left\langle\left(T w, w_{1}\right)\right\rangle+\left\langle\left(f, w_{1}\right)\right\rangle, \\
\left\langle\left(-A w_{2}, w_{2}\right)\right\rangle=\left\langle\left(T w, w_{2}\right)\right\rangle=-\left\langle\left(T w, w_{1}\right)\right\rangle .
\end{gathered}
$$

Now $\left(-A w_{i}, w_{i}\right) \geqslant 0$ may be regarded as the dissipation of the component $w_{i}$, whereas $\left(T w, w_{i}\right)$ is the energy transferred by the advection to that component. Therefore the mean energy transferred to the noninjective scales is positive and identical to the mean dissipation of them, and also equal to the energy lost by the injective scales. Notice that this does not yield any information about if the energy goes to larger or smaller scales than the injective range, or more likely to both. If we decompose again $w_{2}$ in $w_{2}^{+}+w_{2}^{-}$, formed respectively by smaller scales (projection in the $e_{j}: j>l$ ) and larger (projection in $e_{i}: i<k$ ), what we can deduce is

$$
\left\langle\left(T w, w_{2}^{+}\right)\right\rangle+\left\langle\left(T w, w_{2}^{-}\right)\right\rangle=\left\langle\left(-A w_{2}, w_{2}\right)\right\rangle=\left\langle\left(-A w_{2}^{+}, w_{2}^{+}\right)\right\rangle+\left\langle\left(-A w_{2}^{-}, w_{2}^{-}\right)\right\rangle .
$$

Cascades are not merely energy transfer. In the Kolmogorov ${ }^{2}$ theory of homogeneous turbulence, it is admitted that there exists a range of scales (called inertial) where energy is transferred without viscous loss. To prove something similar we need to make an additional hypothesis, also made in Ref. 3: we assume that there is a range outside the injection one such that the projection of $w$ there is zero, or very small. Take the injection range generated by $e_{k}, \ldots, e_{l}$, and assume that from $e_{l}$ to $e_{n}$ there is no projection of $w$. Let $E_{h}^{+}$be the energy transferred to modes larger than $e_{h}$, i.e., the product of $T w$ with the projection $w_{h}^{+}=\sum_{j>h}\left(w, e_{j}\right) e_{j}$. Then 


$$
\left\langle\left(-A w_{2}^{+}, w_{2}^{+}\right)\right\rangle=E_{l+1}^{+}=E_{l+2}^{+}=\cdots=E_{n}^{+}=\left\langle\left(T w, w_{2}^{+}\right)\right\rangle .
$$

Thus the energy passes without loss through this inertial range. Since it goes to smaller scales, it is called a direct cascade. The same could be done for larger scales if we assume that the projection of $w$ in some range $e_{i}, e_{i+1}, \ldots, e_{k-1}$ is zero: then, with an obvious notation,

$$
\left\langle\left(-A w_{2}^{-}, w_{2}^{-}\right)\right\rangle=E_{k-1}^{-}=E_{k-2}^{-}=\cdots=E_{i}^{-}=\left\langle\left(T w, w_{2}^{-}\right)\right\rangle .
$$

This should be an inverse cascade. For the direct one, however, there is a more manageable criterion to ascertain that the projection of $w$ in small ranges is itself small: if the energy satisfies the inequality

$$
\|w\|^{2}=\sum\left|\left(w, e_{n}\right)\right|^{2} \ll \sum \lambda_{n}\left|\left(w, e_{n}\right)\right|^{2}=(-A w, w),
$$

it is because large $\lambda_{n}$ must play the main role, i.e., $w$ is localized at the higher frequencies. Thus a much larger "enstrophy" $(-A w, w)$ than energy means that a direct cascade is more likely, as stated in Ref. 3. This, however, does not seem to be a prerequisite for the actual cascades observed in turbulent phenomena.

\section{EXAMPLES}

\section{A. The Navier-Stokes equations}

The original example, developed as stated in much more depth and detail in Ref. 1, is the Navier-Stokes system for incompressible Newtonian fluids. It is worth to study how the abstract framework applies to this classical case. The Navier-Stokes equations are

$$
\begin{gathered}
\frac{\partial \mathbf{u}}{\partial t}=\nu \Delta \mathbf{u}-\mathbf{u} \cdot \nabla \mathbf{u}-\nabla p+\mathbf{f}, \\
\nabla \cdot \mathbf{u}=0,
\end{gathered}
$$

where $\mathbf{u}$ represents the fluid velocity, $\nu$ the viscosity, $p$ the kinetic pressure and $\mathbf{f}$ the forcing. Boundary conditions determine the space $H$. By taking the divergence of the first equation, one finds that $p$ is in fact a (nonlocal) function of the velocity gradient, the solution of an elliptic problem (see, e.g., Ref. 1). We may either define

$$
\begin{gathered}
T \mathbf{u}=-\mathbf{u} \cdot \nabla \mathbf{u}+\nabla p(\mathbf{u}), \\
A=\nu \Delta,
\end{gathered}
$$

or, as usual, project (18) into the space of functions with null divergence. Denoting by $P$ this projection, one gets the Stokes system:

$$
\frac{\partial \mathbf{u}}{\partial t}=\nu P \Delta \mathbf{u}-P \mathbf{u} \cdot \nabla \mathbf{u}+P \mathbf{f} .
$$

Then we may define $T \mathbf{u}=-P \mathbf{u} \cdot \nabla \mathbf{u}, A=\nu P \Delta$. The space $H$ is defined by the boundary conditions. For periodic ones in a box $\Omega$, one sets

$$
H=\left\{\mathbf{u} \in L^{2}(\Omega)^{N}: \nabla \cdot \mathbf{u}=0, \int_{\Omega} \mathbf{u} \mathrm{d} V=\mathbf{0},\left.\mathbf{u} \cdot \mathbf{n}\right|_{\partial \Omega} \text { antiperiodic }\right\} .
$$


$N$ is the space dimension. The condition $\nabla \cdot \mathbf{u}=0$ is to be understood in the sense of distributions. For these functions the trace of $\mathbf{u} \cdot \mathbf{n}$ at the boundary makes sense. The domain of $A$ is defined as $D(A)=H^{2}(\Omega)^{N} \cap H$.

For no-slip boundary conditions, the velocity at the boundary of the smooth bounded domain $\Omega$ is taken as zero: we therefore set

$$
\begin{gathered}
H=\left\{\mathbf{u} \in L^{2}(\Omega)^{N}: \nabla \cdot \mathbf{u}=0,\left.\mathbf{u} \cdot \mathbf{n}\right|_{\partial \Omega}=0\right\}, \\
D(A)=H^{2}(\Omega)^{N} \cap H_{0}^{1}(\Omega)^{N} \cap H .
\end{gathered}
$$

That $T$ and $A$ satisfy the previous conditions is classical (see, e.g., Ref. 1 or 4).

\section{B. Magnetohydrodynamics}

The MHD system for an incompressible plasma with velocity $\mathbf{u}$ and magnetic field $\mathbf{B}$ are

$$
\begin{gathered}
\frac{\partial \mathbf{u}}{\partial t}=\nu \Delta \mathbf{u}-\mathbf{u} \cdot \nabla \mathbf{u}+\mathbf{B} \cdot \nabla \mathbf{B}-\nabla\left(p+\frac{B^{2}}{2}\right)+\mathbf{f}_{1}, \\
\frac{\partial \mathbf{B}}{\partial t}=\eta \Delta \mathbf{B}-\mathbf{u} \cdot \nabla \mathbf{B}+\mathbf{B} \cdot \nabla \mathbf{u}+\mathbf{f}_{2}, \\
\nabla \cdot \mathbf{u}=0, \\
\nabla \cdot \mathbf{B}=0 .
\end{gathered}
$$

$\eta>0$ is the plasma resistivity. Again a projection $P$ to the space of fields with null divergence is applied to the equations, obtaining

$$
\begin{aligned}
& \frac{\partial \mathbf{u}}{\partial t}=\nu P \Delta \mathbf{u}-P \mathbf{u} \cdot \nabla \mathbf{u}+P \mathbf{B} \cdot \nabla \mathbf{B}+P \mathbf{f}_{1}, \\
& \frac{\partial \mathbf{B}}{\partial t}=\eta P \Delta \mathbf{B}-P \mathbf{u} \cdot \nabla \mathbf{B}+P \mathbf{B} \cdot \nabla \mathbf{u}+P \mathbf{f}_{2} .
\end{aligned}
$$

Now we define $\mathbf{w}=(\mathbf{u} ; \mathbf{B})$,

$$
\begin{gathered}
T \mathbf{w}=(-P \mathbf{u} \cdot \nabla \mathbf{u}+P \mathbf{B} \cdot \nabla \mathbf{B} ;-P \mathbf{u} \cdot \nabla \mathbf{B}+P \mathbf{B} \cdot \nabla \mathbf{u}), \\
A \mathbf{w}=(\nu P \Delta \mathbf{u} ; \eta P \Delta \mathbf{B}) .
\end{gathered}
$$

The space $H$ again depends on the boundary conditions. It is defined essentially as (21) for periodic problems, with the exception that now both components $\mathbf{u}$ and $\mathbf{B}$ of $\mathbf{w}$ must be periodic. $D(A)$ is defined as $H^{2}(\Omega)^{2 N} \cap H$. For Dirichlet homogeneous problems, the analog of (22) is used; in this case $D(A)=H^{2}(\Omega)^{2 N} \cap H_{0}^{1}(\Omega)^{2 N} \cap H$. For the case where the boundary of $\Omega$ is supposed to be a perfect conductor, i.e., $\left.\mathbf{u}\right|_{\partial \Omega}=\mathbf{0},\left.\mathbf{B} \cdot \mathbf{n}\right|_{\partial \Omega}=0,(\nabla \times \mathbf{B}) \times\left.\mathbf{n}\right|_{\partial \Omega}=\mathbf{0}$, one sets

$$
\begin{gathered}
H=\left\{(\mathbf{u} ; \mathbf{B}) \in L^{2}(\Omega)^{2 N}: \nabla \cdot \mathbf{u}=\nabla \cdot \mathbf{B}=0,\left.\mathbf{u} \cdot \mathbf{n}\right|_{\partial \Omega}=\left.\mathbf{B} \cdot \mathbf{n}\right|_{\partial \Omega}=0\right\}, \\
D(A)=\left\{(\mathbf{u} ; \mathbf{B}) \in H^{2}(\Omega)^{2 N} \cap H: \mathbf{u} \in H_{0}^{1}(\Omega)^{N},(\nabla \times \mathbf{B}) \times\left.\mathbf{n}\right|_{\partial \Omega}=\mathbf{0}\right\} .
\end{gathered}
$$

(See, e.g., Refs. 4, 5.) Again $A$ and $T$ satisfy the main conditions and therefore one should expect an analogous transfer of energy for MHD problems. However, it is known that cascades in MHD are very different from the hydrodynamic ones (see, e.g., Ref. 6), which is a warning not to expect fine details from our calculations on energy transfer. 


\section{Passive scalars}

These are magnitudes $\phi$ that are transported and diffused by an incompressible flow with a given velocity $\mathbf{u}$. They evolve according to

$$
\frac{\partial \phi}{\partial t}=\kappa \Delta \phi-\mathbf{u} \cdot \nabla \phi+f
$$

The velocity is a datum of the problem. It satisfies $\nabla \cdot \mathbf{u}=0,\left.\mathbf{u} \cdot \mathbf{n}\right|_{\partial \Omega}=0 . \kappa>0$ is a diffusion coefficient; as we see $\phi$ has no influence on $\mathbf{u}$. Boundary conditions depend on the meaning of $\phi$, and these define the domain of $A=\kappa \Delta$. Set $H=L^{2}(\Omega)$; for Dirichlet problems, $D(A)$ $=H^{2}(\Omega) \cap H_{0}^{1}(\Omega)$; for periodic ones, $D(A)=\left\{\phi \in H^{2}(\Omega)\right.$ : $\left.\phi\right|_{\partial \Omega}$ periodic $\}$; for Neumann ones, $D(A)=\left\{\phi \in H^{2}(\Omega): \partial \phi / \partial n \in H_{0}^{1}(\Omega)\right\}$. The condition of symmetry for $A$,

$$
\int_{\partial \Omega} \phi \frac{\partial \phi}{\partial n} \mathrm{~d} \sigma=0
$$

is satisfied for all $\phi \in D(A)$. Tracers in a fluid are assumed to behave as passive scalars, provided they are not dense enough to modify the density or other properties of the fluid. For instance, salt in ocean water is not taken as a passive, but as an active scalar.

\section{Active scalars}

The equation is formally similar to (27), but now $\mathbf{u}$ depends on $\phi$ through some other equation, making $\mathbf{u}$ a (usually nonlocal) function of $\phi$. These equations are rather common in fluid mechanics (see, e.g., Ref. 7). We will consider two examples: the magnetic potential in twodimensional MHD and the temperature in Bénard convection.

\section{E. Magnetic potential}

Two-dimensional magnetic fields $\mathbf{B}=\left(B_{1}, B_{2}\right)$ in a simply connected domain $\Omega$ are of the form $B_{1}=\partial A / \partial x_{2}, B_{2}=-\partial A / \partial x_{1}$ for some scalar field $A$, called the magnetic potential. With this variable the MHD equations (without forcing on the magnetic field) become

$$
\begin{gathered}
\frac{\partial A}{\partial t}=\eta \Delta A-\mathbf{u} \cdot \nabla A+C(t), \\
\frac{\partial \mathbf{u}}{\partial t}=\nu \Delta \mathbf{u}-\mathbf{u} \cdot \nabla \mathbf{u}+\Delta A \nabla A+f, \\
\nabla \cdot \mathbf{u}=0 .
\end{gathered}
$$

$C(t)$ is some time-dependent constant depending on the choosing of $A$, which is indifferent to the addition of any gradient. Boundary conditions and the gauge constant $C$ are linked. We may choose $C=0$, but at the expense of not being able to precise the values of $A$ at any point. If $\left.\mathbf{B} \cdot \mathbf{n}\right|_{\partial \Omega}=0, A$ is constant along every connected component of $\partial \Omega$. By allowing $C \neq 0$ and choosing $A=0$ at a given point of $\partial \Omega$, we have $\left.A\right|_{\partial \Omega}=0$. Thus we may take $H=L^{2}(\Omega), A=\eta \Delta$, $D(A)=H^{2}(\Omega) \cap H_{0}^{1}(\Omega)$. The second equation of (28) is ignored; we may study the transfer of energy without knowing the full evolution of the system. The magnetic potential is reputed to possess an inverse cascade, ${ }^{6}$ such as the velocity in two-dimensional turbulence. 


\section{F. Temperature in Bénard convection}

In a simple model, this satisfies

$$
\begin{gathered}
\frac{\partial T}{\partial t}=\kappa \Delta T-\mathbf{u} \cdot \nabla T+f, \\
\frac{\partial \mathbf{u}}{\partial t}=\nu \Delta \mathbf{u}-\mathbf{u} \cdot \nabla \mathbf{u}-\nabla p+\mathbf{e}_{2}\left(T-T_{1}\right), \\
\nabla \cdot \mathbf{u}=0 .
\end{gathered}
$$

$T$ is the temperature in a box $[0,1] \times[0,1], \kappa$ is a thermal diffusion constant, $\mathbf{e}_{2}=(1,0), T_{1}$ is the temperature at the upper lid $x_{2}=1$, and $T_{1}+1$ is the temperature at the lower one $x_{2}=0 . p, \mathbf{u}, T$, $\partial \mathbf{u} / \partial x_{1}$ and $\partial T / \partial x_{1}$ are assumed one-periodic in the $x_{1}$-direction, $\mathbf{u}=\mathbf{0}$ at $x_{2}=0$ and $x_{2}=1{ }^{4,8} f$ is a possible injection of temperature.

These conditions make it possible to find $\mathbf{u}$ as a nonlocal function of $T$ (since $\mathbf{u}$ satisfies a parabolic equation on a cylinder) so that we may define for the temperature the spaces

$$
H=L^{2}[0,1] \times L^{2}[0,1]
$$

$$
\begin{aligned}
D(A) & =D(\kappa \Delta) \\
& =\left\{T \in H^{2}(\Omega): T(x, 1)=T_{1}, T(x, 0)=T_{1}+1, T(0, y)=T(1, y), \frac{\partial T}{\partial x_{1}}(0, y)=\frac{\partial T}{\partial x_{1}}(1, y)\right\} .
\end{aligned}
$$

Neumann conditions on the lateral walls are also admissible. Conditions are easily seen to be satisfied (the integral at the boundary of $T \partial T / \partial n$ always vanishes) so that we may ignore $\mathbf{u}$ to see that the temperature is transferred to the different scales according to our model.

\section{CONCLUSIONS}

It is found that the transfer of energy among the different scales acts in a similar way in several processes of fluid mechanics, including the Navier-Stokes equations, the magnetohydrodynamics system, and passive and active scalar equations. The essence of this fact is that these magnitudes follow an evolution equation formed by the addition of a linear dissipative term which determines the scales of the flow and an advective one which distributes the energy injected by the forcing among the different scales. In some extreme cases the presence of a direct or inverse cascade, where energy is transferred without dissipative loss through some inertial range, may also be proved. However, it is pointed that these results do not yield precise information on the transfer of energy in some specific direction, which is known to differ according to the magnitude and the space dimension.

\section{ACKNOWLEDGMENT}

This work was partially supported by the Ministry of Science of Spain under Grant No. BMF2000-0814.

\footnotetext{
${ }^{1}$ C. Foias, O. Manley, R. Rosa, and R. Temam, "Navier-Stokes equations and turbulence," in Encyclopedia of Mathematics and its Applications, Vol. 83 (Cambridge University Press, Cambridge, 2001).

${ }^{2}$ U. Frisch, Turbulence (Cambridge University Press, Cambridge, 1995).

${ }^{3}$ C. Foias, O. Manley, R. Rosa, and R. Temam, C. R. Acad. Sci. Paris, Sér. I 333, 499 (2001).

${ }^{4} \mathrm{R}$. Temam, Infinite-Dimensional Dynamical systems in Mechanics and Physics (Springer, New York, 1988).
} 
${ }^{5}$ M. Sermange and R. Temam, Commun. Pure Appl. Math. 36, 635 (1983).

${ }^{6}$ D. Biskamp, Nonlinear Magnetohydrodynamics (Cambridge University Press, Cambridge, 1993).

${ }^{7}$ P. Constantin, SIAM Rev. 36, 73 (1994).

${ }^{8}$ P. Constantin, Commun. Math. Phys. 129, 241 (1990). 